\title{
Supercontinuum generation in an on-chip silica waveguide
}

\author{
Dong Yoon Oh, ${ }^{1}$ David Sell, ${ }^{1}$ Hansuek Lee, ${ }^{1}$ Ki Youl Yang, ${ }^{1}$ Scott A. Diddams, ${ }^{2}$ and Kerry J. Vahala ${ }^{1, *}$ \\ ${ }^{1}$ T. J. Watson Laboratory of Applied Physics, California Institute of Technology, Pasadena, California 91125, USA \\ ${ }^{2}$ Time and Frequency Division, National Institute of Standards and Technology, Boulder, Colorado 80305, USA \\ *Corresponding author: vahala@caltech.edu
}

Received November 27, 2013; revised January 10, 2014; accepted January 11, 2014; posted January 14, 2014 (Doc. ID 202054); published February 13, 2014

\begin{abstract}
Supercontinuum generation is demonstrated in an on-chip silica spiral waveguide by launching 180 fs pulses from an optical parametric oscillator at the center wavelength of $1330 \mathrm{~nm}$. With a coupled pulse energy of $2.17 \mathrm{~nJ}$, the broadest spectrum in the fundamental TM mode extends from 936 to $1888 \mathrm{~nm}(162 \mathrm{THz})$ at $-50 \mathrm{~dB}$ from peak. There is a good agreement between the measured spectrum and a simulation using a generalized nonlinear Schrödinger equation. (C) 2014 Optical Society of America

OCIS codes: (320.6629) Supercontinuum generation; (190.4390) Nonlinear optics, integrated optics.

http://dx.doi.org/10.1364/OL.39.001046
\end{abstract}

A supercontinuum of light is generated when a narrowband pulse undergoes an extreme spectral broadening due to nonlinear optical processes such as self-phase modulation, cross-phase modulation, Raman scattering, soliton fission, dispersive wave generation, four-wave mixing, and self-steepening [1]. Photonic crystal fiber has been remarkably successful in supercontinuum generation (SCG) due to the feasibility of controlling the dispersion and reducing the mode area by engineering its air-hole geometry [2-4]. Interest in SCG has been driven by applications including frequency combs [5, 6$]$, optical coherence tomography [7], and wavelength division multiplexing $[8,9]$. In the past few years, SCG has also been investigated in several chip-based systems, including silicon photonic nanowires [10-12], chalcogenide waveguides $[13,14]$, and silicon nitride $\left(\mathrm{Si}_{3} \mathrm{~N}_{4}\right)$ waveguides $[15,16]$. These chip-based waveguides for SCG will become important elements to expanding the functionality of photonic integrated circuits.

In this Letter, we demonstrate SCG using an on-chip silica $\left(\mathrm{SiO}_{2}\right)$ waveguide. Silica has a relatively small Kerr coefficient $n_{2}$ of $2.6 \times 10^{-20} \mathrm{~m}^{2} \mathrm{~W}^{-1}$ [17], which is 10 times smaller than silicon nitride and more than 100 times smaller than silicon [18]. Compared to other materials, however, silica has a much lower linear loss, a feature central to the success of optical-fiber-based SCG devices. Recently, a chip-based silica waveguide having recordlow optical loss was demonstrated [19]. In addition to its function as an optical delay line, here the possibility of SCG in the waveguide harnessing its nonlinear interaction length is tested.

The silica waveguide used in the experiment has a total physical path length of $3.5 \mathrm{~m}$ and consists of four cascaded sections [see Fig. 1(a)]. Each section contains two interleaved waveguides configured in the form of an Archimedean spiral. For example, a lightwave traveling from left to right would enter the far-left spiral of Fig. 1(a) at the bottom, take a counterclockwise path toward the spiral center, execute a turn on an S-shaped waveguide at the spiral center, and then exit on a clockwise path. A connection waveguide then conveys the lightwave to the next spiral where entry now occurs on a clockwise path. Each spiral has an outer radius of $7.0 \mathrm{~mm}$. The chip size is $2.5 \mathrm{~cm} \times 6.9 \mathrm{~cm}$. The details of the fabrication method and waveguide design principles can be found in $[19,20]$.

To test spectral broadening using the spiral waveguide, an optical parametric oscillator (OPO, Spectra-Physics OPAL) is used to generate an $80 \mathrm{MHz}$ repetition rate and 180 fs long (FWHM) pulse. The peak power of the pulse is controlled by a variable neutral density filter

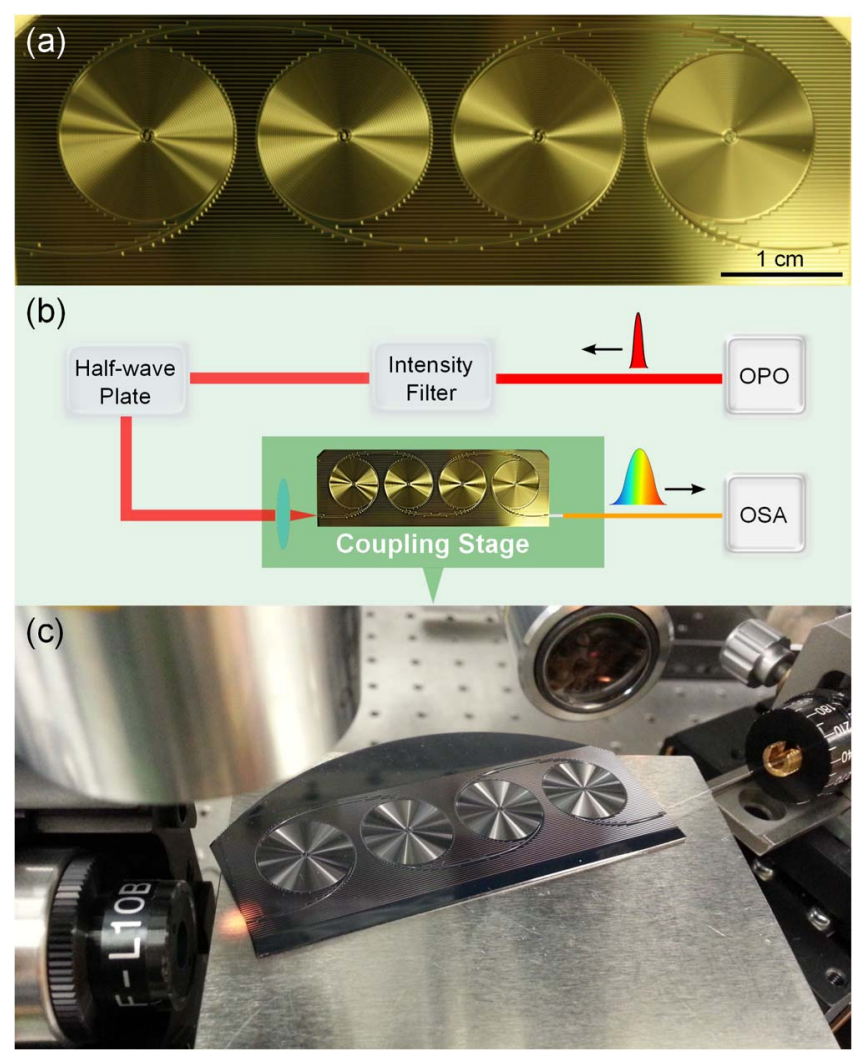

Fig. 1. Low-loss silica-on-silicon waveguide and experimental setup. (a) Photograph of the sample showing four cascaded spiral waveguides. (b) Experimental setup for SCG and characterization using the silica waveguide. OPO, optical parametric oscillator; OSA, optical spectrum analyzer. (c) Close-up view of the optical coupling stage, imaging objectives, and spiral waveguide chip. 
and the polarization by a half-wave plate, as shown in Fig. 1(b). Both TE and TM mode SCG were studied and exhibited qualitatively similar behavior both in the measurements and modeling. However, only data for the TM modes are presented as their spectra are slightly broader on account of a reduced modal, cross-sectional area relative to the TE modes. An objective lens with $12 \mathrm{~mm}$ focal length is mounted on a piezo-controlled $x y z$ stage for the coupling of OPO pulses into the waveguide. Due to the cleave angle of the front facet of the waveguide (introduced to minimize back reflection), the sample is rotated by $35^{\circ}-40^{\circ}$ for efficient coupling. At the end facet, a cleaved multimode fiber is closely positioned to receive the light coming out of the waveguide. The coupling loss at the input and the output ends of the waveguide are estimated to be around 3 and $10 \mathrm{~dB}$, respectively. The other end of the multimode fiber is connected to an OSA. Two OSAs were used to separately record the spectra in the wavelength ranges 600$1700 \mathrm{~nm}$ (Agilent 86141B) and 1200-2400 nm (Yokogawa AQ6375). As the center wavelength of the input pulse is varied from 1250 to $1350 \mathrm{~nm}$, the broadest spectrum is observed near $1330 \mathrm{~nm}$. Measured TM mode spectra for various coupled pulse energies are presented in Fig. 2. At the maximum energy of $2.17 \mathrm{~nJ}$, an octave spanning continuum extending from 936 to $1888 \mathrm{~nm}(162 \mathrm{THz})$ at $-50 \mathrm{~dB}$ from peak is generated.

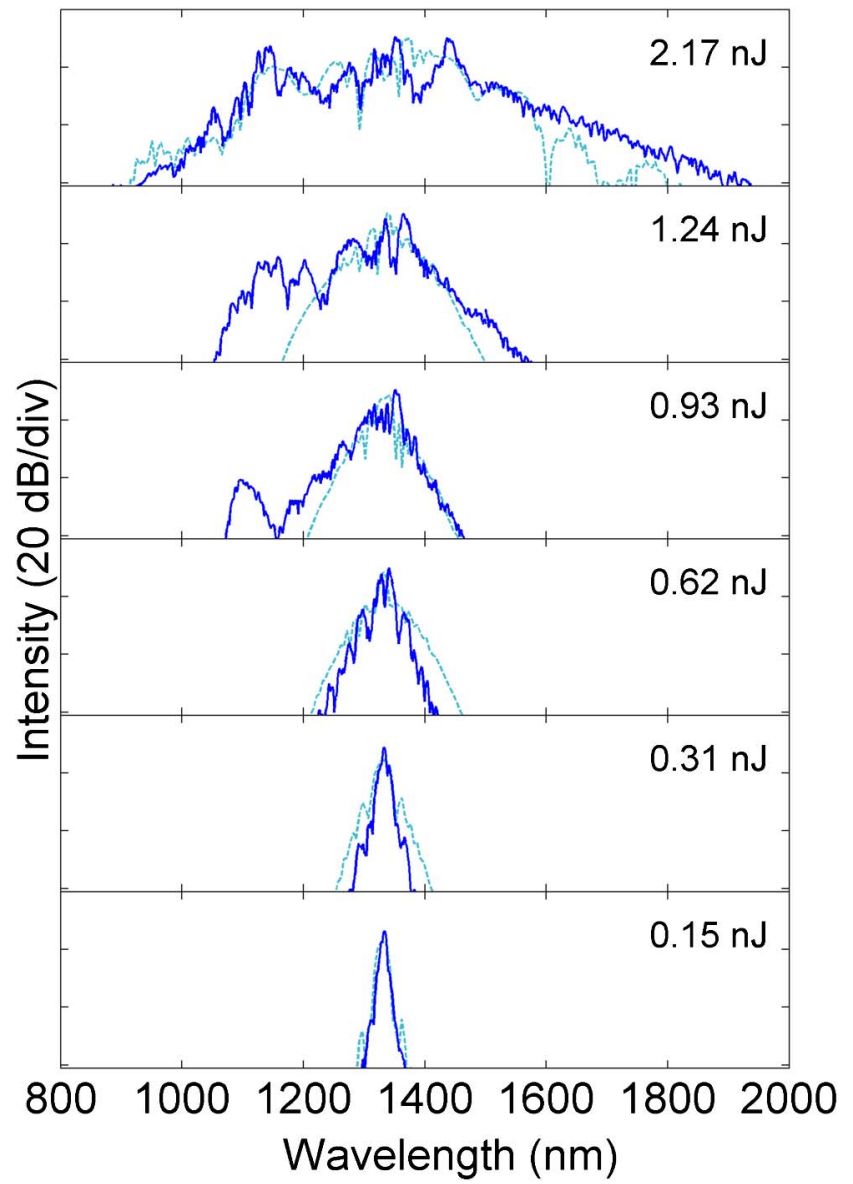

Fig. 2. Spectral broadening in the fundamental TM mode measured at a series of pulse energies. The blue solid curves are measured spectra, and the dashed curves in light blue are spectra from the simulation.
The pulse propagation in the waveguide is modeled by implementing a generalized nonlinear Schrödinger equation [21]:

$$
\begin{aligned}
\frac{\partial \tilde{A}^{\prime}}{\partial z}= & i \gamma(z, \omega) e^{-\mathcal{L}(z, \omega) z} \\
& \times \mathcal{F}\left\{A(z, T) \int_{-\infty}^{\infty} R\left(T^{\prime}\right)\left|A\left(z, T-T^{\prime}\right)\right|^{2} \mathrm{~d} T^{\prime}\right\},
\end{aligned}
$$

$A(z, T)$ is the pulse envelope in a frame of reference moving along the $z$ direction at the group velocity at the pump frequency $\omega_{0}, \tilde{A}\left(z, \omega-\omega_{0}\right)$ is its Fourier transform $(\mathcal{F})$, and $\tilde{A}^{\prime}\left(z, \omega-\omega_{0}\right)$ is defined as $\tilde{A}\left(z, \omega-\omega_{0}\right) e^{-\mathcal{L}(z, \omega) z}$. The function $\mathcal{L}(z, \omega)=\sum_{k=2}^{M}\left(\beta_{k}\left(z, \omega_{0}\right) / k !\right)\left(\omega-\omega_{0}\right)^{k}-$ $(\alpha(z, \omega) / 2)$ has contributions from the dispersion (first term) and the optical loss (second term), where $\beta$ is the propagation constant and $\beta_{k}=\partial^{k} \beta / \partial \omega^{k}$. Once $\beta_{2}(z, \omega)$ is known, then $\beta_{k}\left(z, \omega_{0}\right)$ for $k \geq 3$ can be obtained by fitting $\beta_{2}(z, \omega)$ to a polynomial in $\Delta \omega=\omega-\omega_{0}$. In the simulation, the fitting is taken to 13th order in the summation. Last, $\gamma(z, \omega)$ is the frequency-dependent nonlinearity and $R(T)$ is the nonlinear response function, which has an instantaneous electronic contribution and a contribution from the delayed Raman response [22].

The dispersion, nonlinearity, and scattering loss of the waveguide as functions of waveguide radius in the spiral are calculated using a finite element solver. In Fig. $\underline{3}$, the group velocity dispersion $\left(-\left(2 \pi c / \lambda^{2}\right) \beta_{2}\right)$ and nonlinearity at selected values of waveguide radius $r$ are shown. The outermost part (at $r=7.0 \mathrm{~mm}$ ) of a spiral waveguide has a $8 \mu \mathrm{m}$ thick, $20^{\circ}$ wedge-shaped cross section. It has a zero-dispersion wavelength of $1230 \mathrm{~nm}$ and a nonlinearity of $0.001-0.010 \mathrm{~W}^{-1} \mathrm{~m}^{-1}$ over the wavelength range $600-1700 \mathrm{~nm}$. As the waveguide spirals inside, the zero-dispersion wavelength increases up to $1510 \mathrm{~nm}$ because of the more negative geometric dispersion of the waveguide at smaller radius. Proceeding further inside $(r<0.7 \mathrm{~mm})$, the wave experiences a drop of the zerodispersion wavelength due to a tapering of the waveguide for the minimization of the transition loss over the S-shaped waveguide at the spiral center. At $r=$ $0.2 \mathrm{~mm}$ where the wave enters the S-shaped waveguide that couples light between clockwise and counterclockwise spirals, the waveguide has a triangular cross section with $1.4 \mu \mathrm{m}$ thickness. There, the zero-dispersion wavelength is as short as $720 \mathrm{~nm}$ and nonlinearity is as high as $0.0313-0.145 \mathrm{~W}^{-1} \mathrm{~m}^{-1}$ over the wavelength range $600-1700 \mathrm{~nm}$. Since the length of the tapered section is
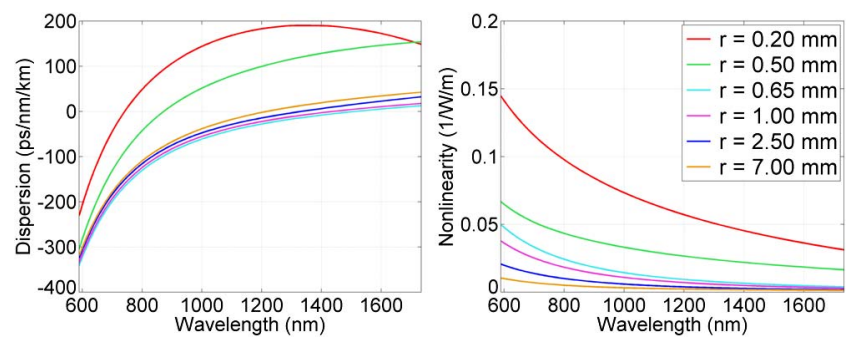

Fig. 3. Calculated dispersion and nonlinearity of the fundamental TM mode at a series of radii that occur at the inner and outer waveguides within each spiral. 
negligible, however, most of the spectral broadening occurs in the nontapered outer region of the spiral.

On account of the radial dependences in Fig. 3, there is a corresponding $z$ dependence for quantities within Eq. (1), which is accounted for as follows. The equation for an Archimedean spiral is $r=b \theta+r_{c}$ in polar coordinates where $r$ is the waveguide radius, $\theta$ is the rotational angle, and the origin is taken to be the spiral center. The parameter $b=0.056 \mathrm{~mm}$ is the distance between successive turnings divided by $2 \pi$, and $r_{c}=0.2 \mathrm{~mm}$ is the radius at which the spiral ends and the S-shaped waveguide starts near the center. Defining $r_{0}=7.0 \mathrm{~mm}$ as the radius at the waveguide entrance $(z=0)$, the radius of the waveguide at the inward propagation length $z$ is $r(z)=\sqrt{r_{0}^{2}-2 b z}$. Outward propagation is modeled in a similar way. Quantities in Eq. (1) are then evaluated at 22 chosen values of radii directly from finite element modeling simulations and interpolated at all the other radii encountered in the waveguide. The resulting spectra from a simulation match well with the measured ones (see Fig. 2).

In conclusion, we have experimentally demonstrated a supercontinuum using an on-chip silica waveguide pumped by femtosecond pulses from an OPO. An octave spanning spectrum in the telecommunication band is generated in the fundamental TM mode. The modeling of the pulse propagation in the spiral waveguide is discussed and a good agreement between the measured spectrum and a simulation is found. With further dispersion engineering and tighter modal confinement, a silica waveguide is expected to be a viable platform for nonlinear optics on a chip.

The authors gratefully acknowledge financial support from the DARPA QuASAR program, NASA, and the Kavli Nanoscience Institute.

\section{References}

1. G. P. Agrawal, Nonlinear Fiber Optics (Academic, 1995).

2. J. K. Ranka, R. S. Windeler, and A. J. Stentz, Opt. Lett. 25, 25 (2000).
3. P. Russell, Science 299, 358 (2003).

4. J. M. Dudley, G. Genty, and S. Coen, Rev. Mod. Phys. 78, 1135 (2006).

5. S. A. Diddams, D. J. Jones, J. Ye, S. T. Cundiff, J. L. Hall, J. K. Ranka, R. S. Windeler, R. Holzwarth, T. Udem, and T. W. Hänsch, Phys. Rev. Lett. 84, 5102 (2000).

6. D. J. Jones, S. A. Diddams, J. K. Ranka, A. Stentz, R. S. Windeler, J. L. Hall, and S. T. Cundiff, Science 288, 635 (2000).

7. I. Hartl, X. D. Li, C. Chudoba, R. K. Ghanta, T. H. Ko, J. G. Fujimoto, J. K. Ranka, and R. S. Windeler, Opt. Lett. 26, 608 (2001).

8. T. Morioka, H. Takara, S. Kawanishi, O. Kamatani, K. Takiguchi, K. Uchiyama, M. Saruwatari, H. Takahashi, M. Yamada, T. Kanamori, and H. Ono, Electron. Lett. 32, 906 (1996).

9. S. Kawanishi, H. Takara, K. Uchiyama, I. Shake, and K. Mori, Electron. Lett. 35, 826 (1999).

10. I. W. Hsieh, X. Chen, X. Liu, J. I. Dadap, N. C. Panoiu, C. Y. Chou, F. Xia, W. M. Green, Y. A. Vlasov, and R. M. Osgood, Opt. Express 15, 15242 (2007).

11. Q. Lin, O. J. Painter, and G. P. Agrawal, Opt. Express 15, 16604 (2007).

12. L. Yin, Q. Lin, and G. P. Agrawal, Opt. Lett. 32, 391 (2007).

13. M. R. Lamont, B. L. Davies, D. Y. Choi, S. Madden, and B. J. Eggleton, Opt. Express 16, 14938 (2008).

14. A. C. Judge, S. A. Dekker, R. Pant, C. M. Sterke, and B. J. Eggleton, Opt. Express 18, 14960 (2010).

15. L. Zhang, Y. Yan, Y. Yue, Q. Lin, O. J. Painter, R. G. Beausoleil, and A. E. Willner, Opt. Express 19, 11584 (2011).

16. R. Halir, Y. Okawachi, J. S. Levy, M. A. Foster, M. Lipson, and A. L. Gaeta, Opt. Lett. 37, 1685 (2012).

17. D. Milam, Appl. Opt. 37, 546 (1998).

18. D. J. Moss, R. Morandotti, A. L. Gaeta, and M. Lipson, Nat. Photonics 7, 597 (2013).

19. H. Lee, T. Chen, J. Li, O. Painter, and K. J. Vahala, Nat. Commun. 3, 867 (2012).

20. T. Chen, H. Lee, J. Li, and K. J. Vahala, Opt. Express 20, 22819 (2012)

21. J. M. Dudley and J. R. Taylor, Supercontinuum Generation in Optical Fibers (Cambridge University, 2010).

22. Q. Lin and G. P. Agrawal, Opt. Lett. 31, 3086 (2006). 